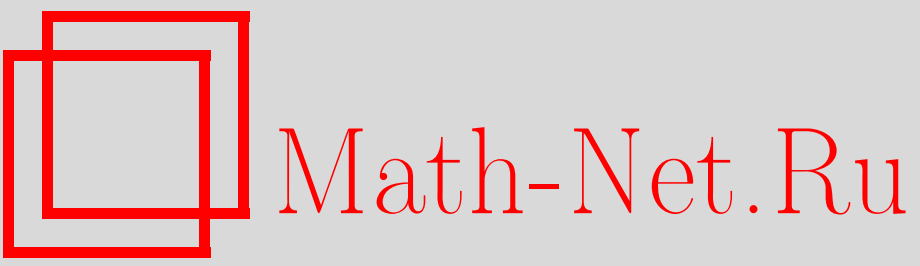

А. В. Михайлов, В. Ю. Новокшенов, Отображение за период для нелинейных импульсов в оптических кабелях с управлением дисперсией, ТМФ, 2003, том 134, номер 1, 124-134

DOI: https://doi.org/10.4213/tmf145

Использование Общероссийского математического портала Math-Net.Ru подразумевает, что вы прочитали и согласны с пользовательским соглашением

http://www . mathnet.ru/rus/agreement

Параметры загрузки:

IP: 54.198 .67 .100

26 апреля 2023 г., 16:24:29 
ТЕОРЕТИЧЕСКАЯ

И МАТЕМАТИЧЕСКАЯ

ФИЗИКА

Том 134, № 1

январь, 2003

(C) 2003 г.

А.В. Михайлов* , В. Ю. Новокшенов ${ }^{\dagger}$

\section{ОТОБРАЖЕНИЕ ЗА ПЕРИОД ДЛЯ НЕЛИНЕЙНЫХ ИМПУЛЬСОВ В ОПТИЧЕСКИХ КАБЕЛЯХ С УПРАВЛЕНИЕМ ДИСПЕРСИЕЙ}

\footnotetext{
Получена простая рекуррентная формула для амплитуды и нелинейного изменения частоты оптического импульса, распространяющегося по кабелю с управлением дисперсией при нулевой средней дисперсии. В пренебрежении диссипацией предполагается, что дисперсия постоянна и имеет противоположные знаки на соседних участках волновода, чем обеспечивается применимость интегрируемых моделей, основанных на нелинейном уравнении Шредингера, в пределах каждого участка. Хорошо известные асимптотические формулы для несолитонных начальных импульсов применимы при выборе достаточно длинных участков для обеспечения формирования самоподобного профиля. Сшивка асимптотических выражений на границах раздела участков дает рекуррентные формулы для амплитуды импульса и нелинейного изменения частоты. Полученные аналитические результаты подтверждаются численным моделированием.
}

Ключевые слова: нелинейное уравнение Шредингера, управление дисперсией, УД-солитон, метод обратной задачи, асимптотика при больших временах.

\section{1. ВВЕДЕНИЕ}

Мы рассматриваем задачу распространения нелинейных электромагнитных импульсов в оптических кабелях с периодическим образом перемежающейся дисперсией (так называемые кабели с управлением дисперсией (УД)) в отсутствие диссипации. Если средняя по периоду дисперсия равна нулю и амплитуда импульса мала, то в соответствии с линейной теорией должна достигаться полная компенсация хроматического уширения. В этом случае отображение за период УД-кабеля должно быть тождественным преобразованием и импульсы должны полностью восстанавливать свои профиили. Однако для обеспечения прохождения импульса с малой ошибкой и для повьшения отношения сигнал/шум необходимо использовать оптические импульсы с относительно высокими амплитудами, так что нелинейные эффекты становятся важными и должны учитываться при теоретическом описании (см. [1]).

*University of Leeds, Leeds, UK; Институт теоретической физики им. Л. Д. Ландау РАН, Черноголовка, Россия

${ }^{\dagger}$ Институт математики Уфимского научного центра РАН, Уфа, Россия 
Решение сформулированной выше задачи представляет собой серьезное достижение с теоретической точки зрения и имеет много важных применений, включая промышленные (см., например, [2]). Множество интересных результатов в этом направлении было получено численно [3]. По-видимому, самые лучшие аналитические результаты были получены с использованием аппроксимации Габитова-Турицына [4].

Наш подход к задаче совершенно иной. Мы используем тот факт, что соответствующие нелинейные уравнения Шредингера (НШ) с положительной и отрицательной дисперсией являются полностью интегрируемыми системами. Схема интегрирования (обратная задача рассеяния, см. [5]), впервые развитая Захаровым и Шабатом в 1971 г. дает адекватные средства для подробного наблюдения за динамикой импульсов. Мы также предполагаем, что длина $z_{0}$ каждого участка кабеля достаточно велика, что позволяет нам использовать асимптотические методы для решения прямой и обратной задач рассеяния (ср. [6]). Нашей целью является нахождение явного отображения импульса за период УД-кабеля. Предположим, что имеется импульс с профилем $\hat{u}_{n}(t)$ на входе $n$-го участка (например, с отрицательной дисперсией), и мы хотим найти профиль $\hat{u}_{n+1}(t)$ импульса на выходе следующего за ним участка с положительной дисперсией. Мы предлагаем следующую схему.

Шаг 1. Используя оператор Лакса $L^{-}$, соответствуюший уравнению НШ с отрицательной дисперсией с потенциалом $u_{n}^{-}\left(t,-z_{0} / 2\right)=\hat{u}_{n}(t)$, мы решаем прямую задачу для определения данных рассеяния.

Шаг 2. Данные рассеяния подчиняются простому эволюционному закону, так что легко найти соответствуюшие данные на выходе $n$-го участка с отрицательной дисперсией.

ШІаг 3. Решая обратную задачу рассеяния, мы находим профиль $u_{n}^{-}\left(t, z_{0} / 2\right)$ на выходе $n$-го участка, который представляет собой начальные данные для следуюшего участка (с положительной дисперсией).

Шаг 4. Используя оператор Лакса $L^{+}$, соответствующий уравнению НШ с положительной дисперсией с потенциалом $u_{n}^{+}\left(t,-z_{0} / 2\right)=u_{n}^{-}\left(t, z_{0} / 2\right)$, мы решаем прямую задачу для определения данных рассеяния.

ШІаг 5. Находим соответствующие данные рассеяния на выходе участка с положительной дисперсией.

Шаг 6. Решаем обратную задачу для нахождения возникаюшего импульса $\hat{u}_{n+1}(t)=$ $u_{n}^{+}\left(t, z_{0} / 2\right)$ после прохождения периода УД-кабеля.

Подчеркнем, что все указанные выше шаги могут быть выполнены явно и, кроме того, мы можем полностью контролировать точность асимптотических решений прямой и обратной задач рассеяния. В результате получаем явное рекуррентное соотношение, которое позволяет найти распространение импульса через многие периоды УД-кабеля.

В отличие от нашей краткой работы по данному вопросу [7], здесь мы предлагаем универсальный подход, включаюший все возможные решения, претендующие на сходимость к УД-солитонам. Таким образом, мы не задаем конкретную фазовую структуру ни потенциалов, ни связанных с ними $\Psi$-функций, ограничивая их только естественной комбинацией квадратичных по времени членов и нелинейным изменением частоты. Это 
дает обобшенное отображение за период, показываюшее, каким образом УД-солитон формируется как стационарная точка итерационного процесса по последовательньм периодам. Его также можно рассматривать как дискретную нелинейную систему для последовательных амплитуд и фаз, которая должна унаследовать некоторые свойства интегрируемости от исходных НШ-моделей с положительной и отрицательной дисперсией.

Сравнение полученного нами аналитического решения с результатами прямого численного моделирования задачи показало их хорошее согласие. Более того, из нашего решения немедленно следует, что нелинейньй вклад в изменение частоты импульса накапливается с расстоянием. Это наблюдение также прекрасно согласуется с результатами численного моделирования.

\section{2. ОСНОВНОЙ ФОРМАЛИЗМ}

Основная модель для описания оптических волокон дается уравнением НШ, которое после подходяшего масштабного преобразования можно записать в безразмерном виде как

$$
i u_{z} \pm u_{t t}+2|u|^{2} u=0, \quad u=u^{ \pm}(t, z) .
$$

Знаки "плюс" и "минус" отвечают участкам с положительной и отришательной дисперсией, соответственно. Известно, что уравнение НШ является интегрируемым, соответствующие операторы Лакса

$$
L^{ \pm}=\left(\begin{array}{cc}
\partial_{t}+\frac{i \lambda}{2} & \mp i \bar{u} \\
-i u & \partial_{t}-\frac{i \lambda}{2}
\end{array}\right), \quad L^{ \pm} \Psi=0
$$

для спектрального преобразования были найдены Захаровым и Шабатом около тридцати лет назад. Рассмотрим импульсы, быстро убываюшие при $|t| \rightarrow \infty$, и предположим, что спектральная задача $(2.2)$ не имеет дискретных собственных значений. Это наиболее интересная область параметров импульса, обычно используемая в оптических телекоммуникационных системах [2]. Непрерывные спектральные данные приводят к стандартной $z$-эволюшии [5]

$$
\Psi \rightarrow \begin{cases}\left(\begin{array}{c}
0 \\
e^{i \lambda t / 2}
\end{array}\right), & t \rightarrow+\infty, \\
\left(\begin{array}{c}
b^{ \pm}(\lambda) e^{ \pm i \lambda^{2} z} e^{-i \lambda t / 2} \\
a^{ \pm}(\lambda) e^{i \lambda t / 2}
\end{array}\right), & t \rightarrow-\infty,\end{cases}
$$

и удовлетворяют условию унитарности $\left|a^{ \pm}\right|^{2} \pm\left|b^{ \pm}\right|^{2}=1$, причем функции $a^{ \pm}$также аналитичны в верхней полуплоскости $\operatorname{Im} \lambda>0$.

Мы будем полагать, что участки как с положительной, так и с отрицательной дисперсией имеют одинаковую длину $z_{0}$, а $\delta=z_{0}^{-1}$-малый параметр нашей теории, $\delta \ll 1$. Удобно ввести обозначение для малого параметра $\varepsilon=z_{0}^{-1} \ln z_{0}$. 
Предположим, что имеется импульс $u(t, 0)$, заданный на середине участка с положительной дисперсией, и что эти начальные данные не содержат солитонов. Тогда на выходе данного участка, т.е. при $z=z_{0} / 2$, импульс асимптотически выражается следующим образом (см. [8], [9]):

$$
u\left(t, \frac{z_{0}}{2}\right)=\delta^{1 / 2}\left[\alpha(\xi)-\varepsilon \frac{\left(\alpha^{4}(\xi)\right)^{\prime \prime}}{4 \alpha(\xi)}+O(\delta)\right] \exp \left\{i\left(\frac{\xi^{2}}{2 \delta}+\varepsilon \frac{2 \alpha^{2}(\xi)}{2 \delta}+O(1)\right)\right\},
$$

где штрихом обозначена производная по $\xi(d / d \xi), \quad \xi=x \delta, \quad \varepsilon=-\delta \ln \delta$, а амплитуду $\alpha(\xi)$ можно выразить в терминах данных рассеяния для соответствуюшей спектральной задачи:

$$
\alpha^{2}(\xi)=-\frac{1}{\pi} \ln \left|a^{+}(\xi)\right|
$$

участок с отрицательной дисперсией

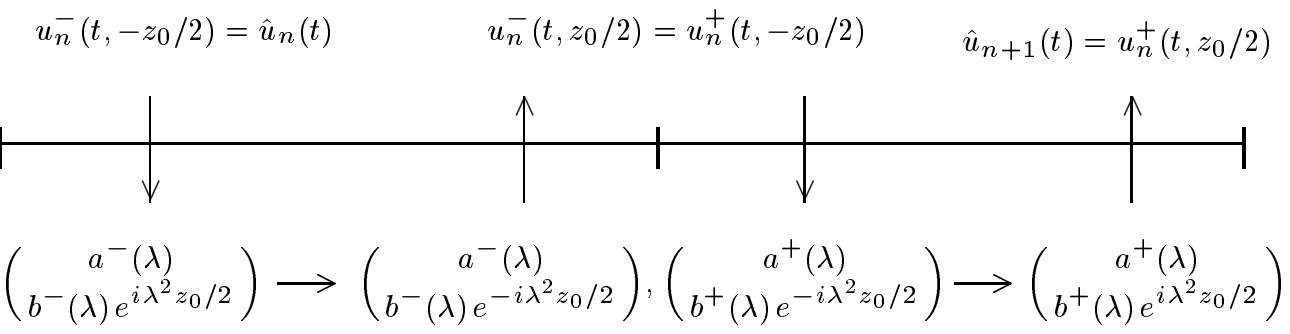

Решение (2.4) подсказывает следующий вид функций $u_{n}^{-}\left(t,-z_{0} / 2\right), u_{n}^{-}\left(t, z_{0} / 2\right)=$ $u_{n}^{+}\left(t,-z_{0} / 2\right)$ и $u_{n}^{+}\left(t, z_{0} / 2\right)$ (см. рисунок):

$$
\begin{aligned}
u_{n}^{-}\left(t,-\frac{z_{0}}{2}\right) & =\delta^{1 / 2}\left(A_{n}^{-}(\xi, \varepsilon)+O(\delta)\right) e^{i S_{n}^{-}(\xi, \varepsilon) / \delta} \\
u_{n}^{-}\left(t, \frac{z_{0}}{2}\right) & =u_{n}^{+}\left(t,-\frac{z_{0}}{2}\right)=\delta^{1 / 2}\left(A_{n}(\xi, \varepsilon)+O(\delta)\right) e^{i S_{n}(\xi, \varepsilon) / \delta} \\
u_{n}^{+}\left(t, \frac{z_{0}}{2}\right) & =\delta^{1 / 2}\left(A_{n}^{+}(\xi, \varepsilon)+O(\delta)\right) e^{i S_{n}^{+}(\xi, \varepsilon) / \delta}
\end{aligned}
$$

где амплитуды и фазы предполагаются конечными относительно $\varepsilon$ :

$$
\left|\frac{d^{k} A_{n}^{ \pm}}{d \xi^{k}}\right|=O(1), \quad\left|\frac{d^{k} S_{n}^{ \pm}}{d \xi^{k}}\right|=O(1), \quad k=0,1,2, \quad \xi \in \mathbb{R}
$$

$$
\left(S_{n}^{+}\right)^{\prime}>0, \quad\left(S_{n}^{-}\right)^{\prime}<0, \quad \xi \in \mathbb{R} .
$$


Представления (2.6)-(2.8) вместе с (2.9), (2.10) определяют класс функций для изучаемых нами решений. Они строго обоснованы с помощью асимптотических решений соответствуюших обратных задач рассеяния, задаюших условия

$$
S_{n}^{ \pm}(\xi, \varepsilon)= \pm \frac{\xi^{2}}{2}+O(\varepsilon) .
$$

Мы называем (2.11) условиями малого нелинейного изменения частоты. Эти условия, возникающие вследствие применения метода Вентцеля-Крамерса-Бриллюэна (ВКБ) для решения соответствующих прямой и обратной задач рассеяния, гарантируют, что вклад в результат дает только одна точка поворота.

\section{3. ПРЯМАЯ СПЕКТРАЛЬНАЯ ЗАДАЧА ДЛЯ УЧАСТКА С ОТРИЦАТЕЛЬНОЙ ДИСПЕРСИЕЙ}

Выполняя шаги 1-6, соответствуюшие изображенным на рисунке, мы будем работать с одним периодом УД-кабеля и опустим индекс $n$ в наших обозначениях, временно полагая

$$
A_{-}=A_{n}^{-}(\xi, \varepsilon), \quad S_{-}=S_{n}^{-}(\xi, \varepsilon) .
$$

Мы восстановим этот индекс в разделе 5 , посвященном рекуррентным соотношениям.

Шаги 1,2 . Для решения прямой задачи рассеяния, соответствующей потенциалу $u_{n}^{-}\left(t,-z_{0} / 2\right)$, подставим выражение (2.6) в спектральную задачу $(2.2)$ и сделаем замену переменных. В результате получим

$$
\begin{aligned}
& \sqrt{\delta} \psi_{1}^{\prime}=-i A_{-} e^{\left(-i S_{-}(\xi, \varepsilon)+i \lambda \xi\right) / \delta} \psi_{2}, \\
& \sqrt{\delta} \psi_{2}^{\prime}=i A_{-} e^{\left(i S_{-}(\xi, \varepsilon)-i \lambda \xi\right) / \delta} \psi_{1},
\end{aligned}
$$

где $\Psi=\left(\psi_{1} e^{i t \lambda / 2}, \psi_{2} e^{-i t \lambda / 2}\right)^{\mathrm{T}}$,

$$
\left(\begin{array}{l}
\psi_{1} \\
\psi_{2}
\end{array}\right) \rightarrow \begin{cases}\left(\begin{array}{l}
0 \\
1
\end{array}\right), & t \rightarrow+\infty \\
\left(\begin{array}{c}
b^{-}\left(\lambda,-\frac{z_{0}}{2}\right) e^{i \lambda^{2} /(2 \delta)} \\
a^{-}\left(\lambda,-\frac{z_{0}}{2}\right)
\end{array}\right), & t \rightarrow-\infty\end{cases}
$$

Система (3.1) является сингулярно-возмущенной вследствие малости параметра при производных. Следуя пионерской работе Захарова и Манакова, мы решаем систему (3.1) методом ВКБ. Стационарные точки (или точки поворота) здесь находятся из уравнения

$$
S_{-}^{\prime}(\xi, 0)-\lambda=0 .
$$

Условие (2.10) гарантирует, что имеется только одна точка поворота. Удобно выбрать следуюшую параметризацию:

$$
\lambda=S_{-}^{\prime}(\mu, 0)=S_{-}(\mu),
$$


где $\mu=\mu(\lambda)$ - спектральный параметр, который единственным образом восстанавливается путем обрашения уравнения (3.4).

В главном порядке уравнения для $\psi_{2}$ система (3.1) дает

$$
i\left(S_{-}^{\prime}-\lambda\right) \psi_{2}^{\prime}+A_{-}^{2} \psi_{2}=-\delta \psi_{2}^{\prime \prime}+\delta A_{-}^{\prime} A_{-}^{-1} \psi_{2}^{\prime} .
$$

Это уравнение имеет следуюшие ВКБ-решения вдали от стационарной точки $\xi=\mu$ :

$$
\begin{aligned}
\psi_{2}(\xi, \mu)= & \exp \left\{\int_{\xi}^{+\infty} \frac{-i A_{-}^{2}(\zeta, \varepsilon)}{S_{-}^{\prime}(\zeta)-S_{-}^{\prime}(\mu)} d \zeta\right\}+O(\sqrt{\delta}), \quad \xi>\mu, \\
\psi_{2}(\xi, \mu)= & a^{-}(\mu, \delta) \exp \left\{\int_{-\infty}^{\xi} \frac{i A_{-}^{2}(\zeta, \varepsilon)}{S_{-}^{\prime}(\zeta)-S_{-}^{\prime}(\mu)} d \zeta\right\}+ \\
& +\sqrt{\delta} b^{-}(\mu, \delta) \frac{A_{-}(\xi, \varepsilon) e^{\left(i S_{-}(\xi, \varepsilon)-i \xi \lambda\right) / \delta}}{S_{-}^{\prime}(\xi)-S_{-}^{\prime}(\mu)} \times \\
& \times \exp \left\{\int_{-\infty}^{\xi} \frac{-i A_{-}^{2}(\zeta, \varepsilon)}{S_{-}^{\prime}(\zeta)-S_{-}^{\prime}(\mu)} d \zeta\right\}+O(\delta), \quad \xi<\mu .
\end{aligned}
$$

Второй член в правой части формулы (3.7) возникает вследствие граничного условия (3.2) для первой компоненты $\psi_{1}$ и второго уравнения (3.1):

$$
\begin{aligned}
& \psi_{1}(\xi, \mu)=O(\sqrt{\delta}), \quad \xi>\mu \\
& \psi_{1}(\xi, \mu)=b^{-}(\mu, \delta) \exp \left\{\int_{-\infty}^{\xi} \frac{-i A_{-}^{2}(\zeta, \varepsilon)}{S_{-}^{\prime}(\zeta)-S_{-}^{\prime}(\mu)} d \zeta\right\}+O(\sqrt{\delta}), \quad \xi<\mu .
\end{aligned}
$$

Сингулярные интегралы в соотношениях (3.9) и (3.6) необходимо продолжить через точку поворота $\xi=\mu$, для того чтобы получить данные рассеяния (3.2). Это можно достигнуть стандартной процедурой сшивки [8].

Вводя переменную

$$
\tau=\delta^{-1 / 2} \sqrt{S_{-}^{\prime \prime}(\mu)}(\xi-\mu)
$$

и используя гладкость $A_{-}$и $S_{-}$, перепишем уравнение (3.5) при $\xi-\mu=O\left(\delta^{1 / 2}\right)$, отбрасывая члены высших порядков:

$$
\frac{d^{2} \psi_{2}}{d \tau^{2}}-i \tau \frac{d \psi_{2}}{d \tau}-\beta(\mu) \psi_{2}=0,
$$

где $\beta(\mu)=A_{-}^{2}(\mu) / S_{-}^{\prime \prime}(\mu)$. Соответствуюшее решение можно выразить в терминах функции параболического цилиндра

$$
\psi_{2}=C(\mu) D_{i \beta}\left(\tau e^{\pi i / 4}\right) e^{i \tau^{2} / 4},
$$

которая имеет следующие асимптотики (см. [10]):

$$
\psi_{2}=C \times \begin{cases}e^{-\pi \beta / 4} \tau^{i \beta}, & \tau \rightarrow+\infty, \\ e^{-\pi \beta / 4} \tau^{i \beta}+\frac{\sqrt{2 \pi} e^{(5 \pi \beta-3 \pi i) / 4}}{\Gamma(-i \beta)} \tau^{-i \beta-1} e^{i \tau^{2} / 2}, & \tau \rightarrow-\infty .\end{cases}
$$

5 Теоретическая и математическая физика, т. 134, № 1, 2003 г. 
Поскольку

$$
\begin{aligned}
& \exp \left\{\int_{\xi}^{+\infty} \frac{-i A_{-}^{2}(\zeta)}{S_{-}^{\prime}(\zeta)-S_{-}^{\prime}(\mu)} d \zeta\right\}=\exp \left\{i \beta(\mu) \ln \left(S_{-}^{\prime}(\xi)-S_{-}^{\prime}(\mu)\right)+\right. \\
& \left.\quad+i \int_{\mu}^{+\infty} \ln \left(S_{-}^{\prime}(\zeta)-S_{-}^{\prime}(\mu)\right) d \beta(\zeta)\right\}=\delta^{i \beta / 2} \tau^{i \beta} M_{1}(\mu)(1+O(\varepsilon)), \quad \xi \rightarrow \mu+0,
\end{aligned}
$$

то сшивка выражений (3.6) и (3.12) достигается при

$$
C(\mu)=\tau^{i \beta(\mu) / 2} e^{\pi \beta(\mu) / 4} M_{1}(\mu),
$$

где

$$
M_{1}(\mu)=\exp \left\{i \int_{\mu}^{+\infty}\left(\frac{A_{-}^{2}(\zeta)}{S_{-}^{\prime \prime}(\zeta)}\right)^{\prime} \ln \left(S_{-}^{\prime}(\zeta)-S_{-}^{\prime}(\mu)\right) d \zeta\right\} .
$$

Разлагая $\psi_{2}$ слева от стационарной точки $\xi=\mu$, из формулы (3.6) получаем

$$
\begin{aligned}
\psi_{2}(\xi, \mu)= & a^{-}(\mu, \delta) \delta^{i \beta / 2}|\tau|^{i \beta} M_{2}(\mu)+\sqrt{\delta} \frac{b^{-}(\mu, \delta)}{\xi-\mu} \frac{A_{-}(\mu)}{\sqrt{S_{-}^{\prime \prime}(\mu)}} e^{\left(i S_{-}(\mu)-i \mu S_{-}^{\prime}(\mu)\right) / \delta} \times \\
& \times e^{\left(i S_{-}^{\prime \prime}(\mu)(\xi-\mu)^{2}\right) /(2 \delta)} \exp \left\{\int_{-\infty}^{\xi} \frac{-i A_{-}^{2}(\zeta, \varepsilon)}{S_{-}^{\prime}(\zeta)-S_{-}^{\prime}(\mu)} d \zeta\right\}+O(\delta)= \\
= & a^{-}(\mu, \delta) \delta^{i \beta / 2}|\tau|^{i \beta} M_{2}(\mu)+\sqrt{\beta} \frac{b^{-}(\mu, \delta)}{\tau} e^{\left(i S_{-}(\mu)-i \mu S_{-}^{\prime}(\mu)\right) / \delta} \times \\
& \times e^{i \tau^{2} / 2} \delta^{-i \beta / 2}|\tau|^{-i \beta} M_{2}^{-1}(\mu)+O(\delta), \quad \xi \rightarrow \mu-0,
\end{aligned}
$$

где

$$
M_{2}(\mu)=\exp \left\{-i \int_{-\infty}^{\mu}\left(\frac{A_{-}^{2}(\zeta)}{S_{-}^{\prime \prime}(\zeta)}\right)^{\prime} \ln \left(S_{-}^{\prime}(\zeta)-S_{-}^{\prime}(\mu)\right) d \zeta\right\} .
$$

Следовательно, условия сшивки выражений (3.12) и (3.6) слева от стационарной точки $(\arg \tau=-\pi)$ имеют вид

$$
\begin{aligned}
C(\mu) e^{-3 \pi \beta(\mu) / 4} & =a^{-}(\mu, \delta) \delta^{i \beta(\mu) / 2} M_{2}(\mu), \\
C(\mu) \frac{\sqrt{2 \pi} e^{(5 \pi \beta-3 \pi i) / 4}}{\Gamma(-i \beta)} & =b^{-}(\mu, \delta) \sqrt{\beta} \delta^{-i \beta(\mu) / 2} e^{\left(i S_{-}(\mu)-i \mu S_{-}^{\prime}(\mu)\right) / \delta} M_{2}^{-1}(\mu) .
\end{aligned}
$$

Окончательно, собирая вместе соотношения (3.13)-(3.15), получаем формулы для спектральных данных в терминах потенциала (2.6):

$$
\begin{gathered}
\left|a^{-}(\lambda)\right|=e^{\pi A_{-}^{2}(\mu) / S_{-}^{\prime \prime}(\mu)}, \quad \lambda=S_{-}^{\prime}(\mu), \quad \operatorname{Im} \lambda=0, \\
\arg a^{-}(\lambda)=\exp \left\{\text { v.p. } \int_{-\infty}^{+\infty} \frac{i A_{-}^{2}(\zeta)}{S_{-}^{\prime}(\zeta)-S_{-}^{\prime}(\mu)} d \zeta\right\}, \\
b^{-}(\lambda)=\exp \left\{\frac{i}{\delta}\left(S_{-}(\mu)-\mu S_{-}^{\prime}(\mu)+\varepsilon \frac{A_{-}^{2}(\mu)}{S_{-}^{\prime \prime}(\mu)}+\delta \phi_{-}(\mu)\right)\right\},
\end{gathered}
$$

где член фазовой поправки $\phi$ имеет вид

$$
\phi_{-}(\mu)=M_{1}(\mu)-M_{2}(\mu)-\frac{3}{4} \pi-\arg \Gamma\left(-\frac{i A_{-}^{2}(\mu)}{S_{-}^{\prime \prime}(\mu)}\right) .
$$




\section{4. ОБРАТНАЯ СПЕКТРАЛЬНАЯ ЗАДАЧА}

При заданных спектральных данных (3.16) и (3.17) вычислим асимптотики потеншиала на следуюшем конце участка с отрицательной дисперсией, т.е. положим $z=z_{0} / 2$. В силу (2.3) спектральные данные теперь имеют вид

$$
a^{-}\left(\lambda, \frac{z_{0}}{2}\right)=a^{-}\left(\lambda,-\frac{z_{0}}{2}\right), \quad b^{-}\left(\lambda, \frac{z_{0}}{2}\right)=b^{-}\left(\lambda,-\frac{z_{0}}{2}\right) e^{-i \lambda^{2} z_{0}} .
$$

Мы будем искать асимптотики потенциала в виде (2.7),

$$
u_{-}\left(t, \frac{z_{0}}{2}\right)=\delta^{1 / 2}(A(\xi, \varepsilon)+O(\delta)) e^{i S(\xi, \varepsilon) / \delta} .
$$

Неизвестные амплитуда $A$ и фаза $S$ находятся путем решения спектральной задачи $(2.2)$, (2.3) с заданными спектральными данными (4.1):

$$
\begin{aligned}
& \sqrt{\tau} \psi_{1}^{\prime}=i A e^{(i S(\xi, \varepsilon)+i \lambda \xi) / \delta} \psi_{2}, \\
& \sqrt{\tau} \psi_{2}^{\prime}=i A e^{(-i S(\xi, \varepsilon)-i \lambda \xi) / \delta} \psi_{1} \text {, } \\
& \left(\begin{array}{l}
\psi_{1} \\
\psi_{2}
\end{array}\right) \rightarrow \begin{cases}\left(\begin{array}{l}
0 \\
1
\end{array}\right), & t \rightarrow+\infty, \\
\left(\begin{array}{c}
b^{-}\left(\lambda,-\frac{z_{0}}{2}\right) e^{-i \lambda^{2} /(2 \delta)} \\
a^{-}\left(\lambda,-\frac{z_{0}}{2}\right)
\end{array}\right), & t \rightarrow-\infty .\end{cases}
\end{aligned}
$$

Таким образом, мы редуцировали обратную задачу рассеяния к прямой, аналогичной той, которую мы решали в разделе 3 . Стационарной точкой теперь является $\lambda=$ $-S^{\prime}(\xi, 0)$, что можно параметризовать как

$$
\lambda=-S^{\prime}(\nu, 0)=-S^{\prime}(\nu),
$$

где $\nu=\nu(\lambda)$ - спектральный параметр, который единственным образом восстанавливается путем обрашения (4.5).

Повторяя явные ВКБ-вычисления для ведуших членов $\psi_{1}, \psi_{2}$, получаем

$$
\begin{gathered}
\psi_{1}(\xi, \nu)=\left\{\begin{array}{l}
O(\sqrt{\delta}), \quad \xi>\nu, \\
b^{-}\left(\lambda,-\frac{z_{0}}{2}\right) e^{-i \lambda^{2} /(2 \delta)} \times \\
\quad \times \exp \left\{\int_{-\infty}^{\xi} \frac{-i A^{2}(\zeta, \varepsilon)}{S_{-}^{\prime}(\zeta)-S^{\prime}(\mu)} d \zeta\right\}+O(\sqrt{\delta}), \quad \xi<\nu,
\end{array}\right. \\
\psi_{2}(\xi, \mu)=\left\{\begin{array}{c}
\exp \left\{\int_{\xi}^{+\infty} \frac{-i A^{2}(\zeta, \varepsilon)}{S^{\prime}(\zeta)-S^{\prime}(\mu)} d \zeta\right\}+O(\sqrt{\delta}), \quad \xi>\nu, \\
a^{-}\left(\lambda,-\frac{z_{0}}{2}\right) \exp \left\{\int_{-\infty}^{\xi} \frac{i A^{2}(\zeta, \varepsilon)}{S^{\prime}(\zeta)-S^{\prime}(\nu)} d \zeta\right\}+ \\
\quad+\sqrt{\delta} b^{-}\left(\lambda,-\frac{z_{0}}{2}\right) e^{-i \lambda^{2} /(2 \delta)} \frac{A(\xi, \varepsilon) e^{(i S(\xi, \varepsilon)-i \xi \lambda) / \delta}}{S^{\prime}(\xi)-S^{\prime}(\nu)} \times \\
\quad \times \exp \left\{\int_{-\infty}^{\xi} \frac{-i A^{2}(\zeta, \varepsilon)}{S^{\prime}(\zeta)-S^{\prime}(\nu)} d \zeta\right\}+O(\delta), \quad \xi<\mu .
\end{array}\right.
\end{gathered}
$$


Разложение вблизи точки поворота $\xi=\nu$ снова выполняется, как и в разделе 3 , с использованием функции параболического цилиндра и сшивки (4.6) и (4.7) с помошью асимптотики (3.12). Окончательно, сшивка дает уравнения для $A$ и $S$ в терминах данных рассеяния (2.7):

$$
\begin{gathered}
\left|a^{-}\left(\lambda,-\frac{z_{0}}{2}\right)\right|=e^{-\pi A^{2}(\nu) / S^{\prime \prime}(\nu)}, \quad \lambda=-S^{\prime}(\nu), \quad \operatorname{Im} \lambda=0, \\
b^{-}\left(\lambda,-\frac{z_{0}}{2}\right)=\exp \left\{\frac{i}{\delta}\left(S(\nu)-\nu S^{\prime}(\nu)-\varepsilon \frac{A^{2}(\nu)}{S^{\prime \prime}(\nu)}+\delta \phi(\nu)\right)\right\},
\end{gathered}
$$

где конечный сдвиг фазы $\phi$ такой же, как в (3.18), при подстановке $A_{-}(\mu) \rightarrow A(\nu)$ и $S_{-}(\mu) \rightarrow S(\nu)$.

Шаг 3. Используя асимптотические решения прямой и обратной задач рассеяния для оператора $L^{-}$, найденного выше в настояшем разделе и в разделе 3 , можно связать амплитуду $A_{-}$и фазу $S_{-}$на выходе участка с отрицательной дисперсией с амплитудой $A$ и фазой $S$ на входе данного участка. Сравнивая данные рассеяния $(3.16),(3.17)$ с (4.8), (4.9), получаем

$$
\begin{gathered}
\frac{A_{-}^{2}(\mu)}{S_{-}^{\prime \prime}(\mu)}=-\frac{A^{2}(\nu)}{S^{\prime \prime}(\nu)}, \quad \lambda=S_{-}^{\prime}(\mu)=-S^{\prime}(\nu), \\
S_{-}(\mu)-\mu S_{-}^{\prime}(\mu)+\varepsilon \frac{A_{-}^{2}(\mu)}{S_{-}^{\prime \prime}(\mu)}=S(\nu)-\nu S^{\prime}(\nu)+\varepsilon \frac{A^{2}(\nu)}{S^{\prime \prime}(\nu)} .
\end{gathered}
$$

Шаги 3-6. Аналогично тому, как это было сделано на шагах 1-3, мы решаем прямую и обратную задачи рассеяния для оператора $L^{+}$на участке с положительной дисперсией. Это позволяет связать амплитуду $A$ и фазу $S$, заданные на входе участка, со значениями $A_{+}$и $S_{+}$на выходе. Результат очень похож на выражения (4.10):

$$
\begin{gathered}
\frac{A^{2}(\nu)}{S^{\prime \prime}(\nu)}=-\frac{A_{+}^{2}(\kappa)}{S_{+}^{\prime \prime}(\kappa)}, \quad \lambda=-S^{\prime}(\nu)=S_{+}^{\prime}(\kappa) \\
S(\nu)-\nu S^{\prime}(\nu)+\varepsilon \frac{A^{2}(\nu)}{S^{\prime \prime}(\nu)}=S_{+}(\kappa)-\kappa S_{+}^{\prime}(\kappa)+\varepsilon \frac{A_{+}^{2}(\kappa)}{S_{+}^{\prime \prime}(\kappa)},
\end{gathered}
$$

где $\operatorname{Im} \lambda=0$.

\section{5. ФОРМУЛЫ ОТОБРАЖЕНИЯ ЗА ПЕРИОД}

Суммируя результаты, полученные в предыдущем разделе, и восстанавливая индекс $n$, который нумерует секции УД-кабеля, мы приходим к рекуррентным соотношениям для амплитуд и логарифмических фаз импульса:

$$
\begin{aligned}
S_{n}^{-}(\mu, \varepsilon) & -\mu \frac{d S_{n}^{-}(\mu, \varepsilon)}{d \mu}+\varepsilon\left(A_{n}^{-}(\mu, \varepsilon)\right)^{2}\left(\frac{d^{2} S_{n}^{-}(\mu, \varepsilon)}{d \mu^{2}}\right)^{-1}= \\
= & S_{n}(\nu, \varepsilon)-\nu \frac{d S_{n}(\nu, \varepsilon)}{d \nu}+\varepsilon\left(A_{n}(\nu, \varepsilon)\right)^{2}\left(\frac{d^{2} S_{n}(\nu, \varepsilon)}{d \nu^{2}}\right)^{-1}
\end{aligned}
$$




$$
\begin{gathered}
\left(A_{n}^{-}(\mu, \varepsilon)\right)^{2}\left(\frac{d^{2} S_{n}^{-}(\mu, \varepsilon)}{d \mu^{2}}\right)^{-1}=-\left(A_{n}(\nu, \varepsilon)\right)^{2}\left(\frac{d^{2} S_{n}(\nu, \varepsilon)}{d \nu^{2}}\right)^{-1} \\
S_{n}(\nu, \varepsilon)-\nu \frac{d S_{n}(\nu, \varepsilon)}{d \nu}+\varepsilon\left(A_{n}(\nu, \varepsilon)\right)^{2}\left(\frac{d^{2} S_{n}(\nu, \varepsilon)}{d \nu^{2}}\right)^{-1}= \\
=S_{n}^{+}(\kappa, \varepsilon)-\kappa \frac{d S_{n}^{+}(\kappa, \varepsilon)}{d \kappa}+\varepsilon\left(A_{n}^{+}(\kappa, \varepsilon)\right)^{2}\left(\frac{d^{2} S_{n}^{+}(\kappa, \varepsilon)}{d \kappa^{2}}\right)^{-1} \\
-\left(A_{n}(\nu, \varepsilon)\right)^{2}\left(\frac{d^{2} S_{n}(\nu, \varepsilon)}{d \nu^{2}}\right)^{-1}=\left(A_{n}^{+}(\kappa, \varepsilon)\right)^{2}\left(\frac{d^{2} S_{n}^{+}(\kappa, \varepsilon)}{d \kappa^{2}}\right)^{-1}
\end{gathered}
$$

Здесь $\mu, \nu$ и $\kappa$-решения уравнений

$$
\xi=\frac{d S_{n}^{-}(\mu, 0)}{d \mu}=-\frac{d S^{-}(\nu, 0)}{d \nu}=\frac{d S_{n}^{+}(\kappa, 0)}{d \kappa} .
$$

Эти решения являются единственными, обеспечиваюшими условия малости нелинейного изменения частоты (2.11).

Из приведенных вьше рекуррентных соотношений следует, что условие малости нелинейного изменения частоты не выполняется равномерно и нарушается на некотором расстоянии, даже если оно достаточно хорошо выполнялось для входного импульса. Действительно, предположим, что начальный профиль импульса имеет вид (2.4), т.е.

$$
S_{1}^{+}(\xi, \varepsilon)=\frac{\xi^{2}}{2}+\varepsilon \alpha^{2}(\xi)+O(1), \quad A_{1}^{+}(\xi, \varepsilon)=\alpha(\xi)-\varepsilon \frac{\left(\alpha^{4}(\xi)\right)^{\prime \prime}}{4 \alpha(\xi)}+O(\delta) .
$$

Из приведенных вьше рекуррентных соотношений следует, что после $n$ периодов распространения мы получим

$$
S_{n+1}^{+}(\xi, \varepsilon) \sim S_{n}^{+}(\xi, \varepsilon)+2 \varepsilon A_{n}^{+}(\xi, \varepsilon) .
$$

Ясно, что при $n \sim \max \left(A_{1}^{1}(\xi)\right) \varepsilon^{-1}$ условие $(2.9)$ не выполняется и мы должны учитывать другие точки поворота в анализе ВКБ, что лежит за рамками настояшей работы. Согласно нашей теории нелинейный вклад в изменение частоты возрастает при распространении импульса вдоль УД-кабеля. Несмотря на это результаты прямого численного моделирования уравнения НШ с перемежающейся дисперсией хорошо согласуются с нашими аналитическими результатами. Мы сравнили полученные в результате численных расчетов профили импульса в точках на границах с аналитическими выражениями, полученными с помощью рекуррентных соотношений. Полученное аналитически возрастание нелинейного вклада в изменение частоты прекрасно согласуется с результатами численного моделирования [2].

В заключение нашего исследования УД-отображений отметим, что оно демонстрирует новый подход к задаче описания распространения импульса в оптических УД-кабелях. Мы полагаем, что это может быть полезным для дальнейшего понимания и даже описания эволюции оптических импульсов на нескольких начальных периодах его распространения в УД-системах. Очень важно, что можно строго контролировать точность (а 
именно, остаточные члены) в наших асимптотических формулах. Условие малости нелинейного изменения частоты (2.11) налагает естественные ограничения на применимость нашей теории. Мы по-прежнему не можем аналитически контролировать формирование настояших УД-солитонов. Имеются значительные сложности в применении асимптотической техники ВКБ при рассмотрении кратных стационарных точек, дающих вклад в решения соответствуюших прямой и обратной задач рассеяния. Работа в этом направлении в настояшее время продолжается. Мы надеемся преодолеть эту проблему и получить функциональную дискретную динамическую систему для соответствующих амплитуд и фаз, в которой УД-солитон появлялся бы в качестве аттрактора. Это также открыло бы перспективу для решения представляюшей значительный интерес задачи описания взаимодействия УД-солитонов.

Другим многообешающим направлением развития является изучение систем с усилителями и фильтрами в точках соединения участков с различными дисперсиями. Легко контролировать динамику импульсов, умножая коэффициент прохождения $a(\lambda)$ на фиксированную константу (коэффициент усиления) или даже на функцию (фильтрация). Можно также очевидным образом модифицировать приведенную выше теорию применительно к УД-системам с любым кусочно-постоянным профилем дисперсии.

Благодарности. Работа выполнена при частичной поддержке РФФИ (проект № 02-01-00144) и INTAS (грант № 99-1782). Один из авторов (В.Ю.Н) благодарит Институт математических наук Исаака Ньютона (Кембридж, Великобритания) за поддержку и гостеприимство. А.В.М. благодарен Королевскому обществу Великобритании за поддержку объединенного проекта с Институтом теоретической физики им. Л. Д. Ландау РАН.

\section{Список литературы}

[1] A. Hasegava. Solitons in Optics. New York: Academic Press, 1995.

[2] Optical Solitons. Theoretical Challenges and Industrial Perspectives. EDP Sciences. Eds. V. E. Zakharov, S. Wabnitz. Berlin: Springer, 1999.

[3] J. H. B. Nijhof, S. K. Turitsyn, N. J. Doran. Dispersion-managed solitons and the inverse scattering transform. In: Nonlinear Guided Waves and their Applications. OSA Technical Digest. Washington: OSA, 1999. P. 313.

[4] I. Gabitov, S. Turitsin. Opt. Lett. 1996. V. 21. P. 327; V. E. Zakharov, S. V. Manakov. Письма в ЖЭТФ. 1999. Т. 70. № 9. С. 573; S. Turitsin, V. Mezentsev. Письма в ЖЭЭФ. 1998. T. 67. C. 616.

[5] В. Е. Захаров, С. В. Манаков, С. П. Новиков, Л. П. Питаевский. Теория солитонов. Метод обратной задачи. М.: Наука, 1980.

[6] В. Е. Захаров, С. В. Манаков. ЖЭТФ. 1976. Т. 71. № 1(7). С. 203.

[7] A. V. Mikhailov, V. Yu. Novokshenov. Письма в ЖЭЭФ. 2001. Т. 73. № 5. С. 287.

[8] В. Ю. Новокшенов. ДАН СССР. 1980. Т. 251. № 4. С. 799; P. A. Deift, X. Zhou. Commun. Math. Phys. 1994. V. 165. P. 175.

[9] A. R. Its, A. G. Izergin, V. E. Korepin, G. G. Varzugin. Physica D. 1992. V. 54. P. 351-395.

[10] Г. Бейтмен, А. Эрдейи. Высшие трансцендентные функции. Т. 2. М.: Наука, 1974. 